\title{
Cross-wavelet analysis of wall pressure fluctuations beneath incompressible turbulent boundary layers
}

\author{
R. CAMUSSI ${ }^{1}$, G. ROBERT ${ }^{2}$ AND M. C. JACOB \\ ${ }^{1}$ Dipartimento di Ingegneria Meccanica e Industriale, Universitá Roma Tre, Via della Vasca Navale 79, \\ 00146 Roma, Italy \\ ${ }^{2}$ Centre Acoustique du LMFA, UMR CNRS 5509, Ecole Centrale de Lyon, Université Claude-Bernard \\ Lyon I, F-69134 Ecully Cedex, France
}

(Received 9 July 2007 and in revised form 24 July 2008)

Pressure fluctuations measured at the wall of a turbulent boundary layer are analysed using a bi-variate continuous wavelet transform. Cross-wavelet analyses of pressure signals obtained from microphone pairs are performed and a novel postprocessing technique aimed at selecting events with strong local-in-time coherence is applied. Probability density functions and conditionally averaged equivalents of Fourier spectral quantities, usually introduced for modelling purposes, are computed. The analysis is conducted for signals obtained at low Mach numbers from two different non-equilibrium turbulent boundary layer experiments. It is found that that the selected events, though statistically independent, exhibit bi-modal statistics while the conditional coherence function coincides with its non-conditional Fourier equivalent. The physical nature of the selected events has been further explored by the computation of ensemble-averaged pressure time signatures and the results have been physically interpreted with the aid of numerical and experimental results from the literature. In both experiments, it has been found that the major physical mechanisms responsible for the observed conditional statistics are represented by sweep-type events which can be ascribed to the effect of streamwise vortices in the near-wall region. More precisely, the wavelet analysis highlights the convection of the selected structures in both cases. Conversely, compressibilty effects could be related to these events only in one case.

\section{Introduction}

Pressure fluctuations induced at the wall by turbulent boundary layers play a major role in many physical problems of engineering interest since they contribute significantly to the generation of surface vibrations and noise radiation. As an example, the design of modern commercial aircraft requires models of the fluctuating wall pressure field in order to predict interior noise generation mechanisms and to assess the lifetime of fuselage panels subjected to fatigue stress. These issues have motivated a large number of studies in the past few decades, most of them devoted to the statistical characterization of the random pressure field at the wall. Experiments and numerical simulations have been carried out, mainly with the purpose of developing semi-empirical models providing reliable predictions of representative statistical wall pressure indicators (see e.g. the review given by Bull 1996). As pointed out by Blake (1986), among various statistical quantities, a significant role is played 
by the coherence function between two signals, which is defined as (Bendat \& Piersol 2000)

$$
\gamma^{2}(\omega)=\frac{\left|S_{12}(\omega)\right|^{2}}{S_{11}(\omega) S_{22}(\omega)}
$$

where $S_{i j}(i=1,2, j=1,2)$ denotes the Fourier auto-(for $\left.i=j\right)$ and cross-spectra (for $i \neq j$ ) and $\omega$ the angular frequency. For example, the Corcos (1963a) model predicts an exponential decay of the coherence function which is in good agreement with experimental and numerical data. Although more sophisticated analytical representations have been proposed in more recent models (see among many the models proposed by Chase 1980; Efimtzov 1986; Smol' yakov \& Tkachenko 1991; and the review by Graham 1997) Corcos' approach is still considered one of the most reliable (see e.g. the recent work by Brungart et al. 2002).

Despite the large body of literature devoted to the study of statistical wall pressure properties, there is still some room for improvement in the field, especially regarding the physical nature of the fluid dynamic events responsible for the observed pressure field statistics. This is an important issue from the practical viewpoint since a deeper knowledge of the fluid dynamic structures underlying the observed pressure properties may be helpful in addressing suitable control strategies aimed at manipulating the flow structures and modifying the wall pressure behaviour.

Numerical simulations of simplified configurations have attempted to clarify the connection between wall pressure fields and near-wall vortical structures whose topology was selected a priori according to classical conceptual models of the turbulent boundary layer. For example, Dhanak \& Dowling (1995) and Dhanak, Dowling \& Si (1997), followed the quasi-two-dimensional conceptual model of Orlandi \& Jimenez (1994), and clarified the effect of near-wall quasi-streamwise structures on the wall pressure field. More recently, Ahn, Graham \& Rizzi (2004) reproduced correlations and spectra at the wall. In order to estimate the wall pressure distribution, they also reproduced hairpin vortex dynamics on the basis of the so-called attached eddy model (Perry \& Chong 1982). Only a few experiments have been focused on these aspects, since the correlation between wall pressure and coherent structures is rather difficult to interpret owing to the chaotic nature of the pressure field. Among the existing studies, the work by Johansson, Her \& Haritonidis (1987) can be mentioned: they carried out simultaneous pressure-velocity measurements and suggested physical mechanisms for the underlying generation of positive or negative pressure peaks at the wall. However, they did not clarify the connection between the educed structures and the wall pressure spectral quantities. More recently, Farabee \& Casarella (1991) proposed a connection between the wall pressure wavenumber spectra and physical quantities describing the turbulent boundary layer. They suggested that the highwavenumber components should be attributed to fluid dynamic structures in the near-wall region while the low-wavenumber domain is influenced by the large-scale structures in the outer layer (see also Bradshaw 1967).

The main objective of the present work is to extend the analysis of experimental data to non-equilibrium boundary layers and to the cross-wavelet approach. This is intended to provide a qualitative picture of the coherent events associated with the most strongly cross-correlated wall pressure events. Available wall pressure data obtained experimentally in two different laboratory flows are analysed and properly post-processed in order to extract the desired information. Owing to the random nature of the pressure signals at the wall, data are statistically treated through conditional analyses. Conditional coherence functions and ensemble-averaged 
pressure time signatures are obtained and results are interpreted with reference to the above-mentioned simplified conceptual models of the turbulent boundary layer. The conditioning procedure proposed herein is based on the selection of events which are determined through the computation of a time-frequency localized equivalent of the Fourier coherence, obtained by the application of the cross-wavelet transform to pairs of signals. More details about the wavelet-based approach are given in the next section. The experimental set-up for each experiment and the flow conditions of the analysed experimental databases are briefly described in $\S 3$ while the main results are presented in $\S 4$ together with the suggested physical interpretations. Final remarks and conclusions are given in $\S 5$.

\section{Post-processing method}

During the last two decades, wavelet analysis has been extensively applied to analyse random data obtained from experimental investigations or numerical simulations of turbulent flows. Comprehensive reviews of the theory and application of wavelets can be found in many reference papers or books (e.g. Mallat 1989; Daubechies 1992; Farge 1992). The wavelet transform of single-point velocity signals has been successfully applied to track coherent structures in turbulent shear flows and to characterize their statistical properties (see e.g. Camussi \& Guj 1997; Guj \& Camussi 1999; Camussi \& Di Felice 2006). Wavelet analyses of wall pressure fluctuations have been carried out by Poggie \& Smiths (1997) and Lee \& Sung (2002). The latter authors showed interesting features of the propagation of the pressure perturbations and their phase velocity.

In the approach adopted in the present study, a wavelet analysis is applied to select events reaching significant coherence levels between two wall pressure signals. For this purpose, a procedure aimed at determining the wavelet equivalents of standard Fourier cross-spectra and coherence functions is applied. From the theoretical viewpoint, a brief discussion about the fundamentals of the cross-wavelet analysis was presented in Torrence \& Compo (1998) though a bi-variate extension of the wavelet transform had already been reported by Hudgins, Friebe \& Mayer (1993). Applications of crosswavelet analysis to turbulent flows or geophysical data series are shown in Onorato et al. (1997), Li (1998), Grinsted, Moore \& Jevrejeva (2004) and Maraun \& Kurths (2004).

Owing to the large body of literature available, we limit ourselves to a brief discussion of the principal features. Considering two signals $p_{1}(t)$ and $p_{2}(t)$ measured simultaneously, e.g. with two microphones, a wavelet transform can be used to select events which are closely correlated on a local, in frequency and time, level. Formally, the wavelet transform of $p_{1}(t)$ at the resolution scale $r$, which is proportional to the inverse of the frequency, is given by the following expression:

$$
w_{1}(r, t)=C_{\Psi}^{-1 / 2} r^{-1 / 2} \int_{\infty}^{\infty} \Psi^{*}\left(\frac{t-\tau}{r}\right) p_{1}(\tau) \mathrm{d} \tau,
$$

where the integral represents a convolution product with the dilated and translated counterpart of the so-called mother wavelet $\Psi(t)$, the asterisk denotes complex conjugation, and $C_{\Psi}^{-1 / 2}$ is a normalization coefficient which accounts for the mean value of $\Psi(t)$. In the approach proposed here, the continuous complex Morlet wavelet kernel has been adopted since it provides a good balance between time and frequency localization.

The Morlet wavelet was originally presented by Goupillaud, Grossman \& Morlet (1984) and has been successively used in many applications (see among many Hudgins 
et al. 1993). The mother wavelet consists of a plane wave modulated by a Gaussian and is defined as follows

$$
\Psi(t)=A \mathrm{e}^{\mathrm{i} \omega_{0} t} \mathrm{e}^{-t^{2} / 2} .
$$

The coefficient $\omega_{0}$ is usually taken equal to 6 in order to minimize errors related to the non-zero mean (see also Onorato et al. 1997).

Equation (2.1) can be applied to the signal $p_{2}(t)$, leading to another set of coefficients $w_{2}(r, t)$. By combining the two sets of coefficients it is possible to define a wavelet cross-scalogram as follows:

$$
w_{12}(r, t)=w_{1}(r, t) w_{2}^{*}(r, t) .
$$

The wavelet coherence is denoted $R^{2}(r, t)$ and it is computed following the procedure proposed by Torrence \& Webster (1998), successively applied by Jevrejeva, Moore \& Grinsted (2003) and Grinsted et al. (2004), and formalized as follows:

$$
R^{2}(r, t)=\frac{\left|\left\langle w_{12}(r, t)\right\rangle\right|^{2}}{\left\langle w_{1}(r, t)\right\rangle^{2}\left\langle w_{2}(r, t)\right\rangle^{2}} .
$$

Hence the wavelet coherence is a normalized scalogram. As suggested by Maraun \& Kurths (2004), it is thus possible to separate peaks due to actual large correlation levels from those due to large amplitude of one or both the original signals in a similar manner as the classical correlation coefficient does for the cross-correlation. In particular, the quantity in (2.4) closely resembles the definition of a traditional correlation coefficient, but localized in the time-frequency space and having an amplitude between 0 and 1 . The square brackets in (2.4) denotes a smoothing operator acting both in time and scale which, through the convolution with proper smoothing functions, recovers the correct statistical definition of the coherence (see Torrence \& Webster 1999, for the details).

Let $S$ be the smoothing function. The smoothing operator acting both upon the time $t$ and the scale $r$, can then be written as follows:

$$
S(w)=S_{\text {scale }}\left(S_{\text {time }}(w(r, t))\right) .
$$

$S_{\text {scale }}$ denotes the smoothing operator along the wavelet resolution axis and $S_{\text {time }}$ the analogous operator along the time axis. As suggested by Torrence \& Webster (1999), in the Morlet wavelet case the smoothing operator is

$$
S_{\text {time }}(w(r, t))=w(r, t) * c_{1} \exp \left(-\frac{t^{2}}{2 r^{2}}\right)
$$

at a fixed resolution $r$, and

$$
S_{\text {scale }}(w(r, t))=w(r, t) * c_{2} \Pi(0.6 r)
$$

at a fixed time $t$. The symbol*denotes the convolution product, $c_{1}$ and $c_{2}$ are normalization coefficients and $\Pi$ is the rectangle function. The smoothing operator is therefore designed to have a footprint similar to that of the wavelet adopted. The scale smoothing is done using a boxcar filter of width 0.6. This factor has been determined in Torrence \& Compo (1998) as the scale decorrelation length for the Morlet wavelet. As was clarified by Torrence \& Webster (1999) the width of the Morlet wavelet function in both time and Fourier space provides a natural width of the smoothing function. In summary the smoothing is done using a weighted running average (or convolution) in both the time and scale directions. It has been checked that modifying 


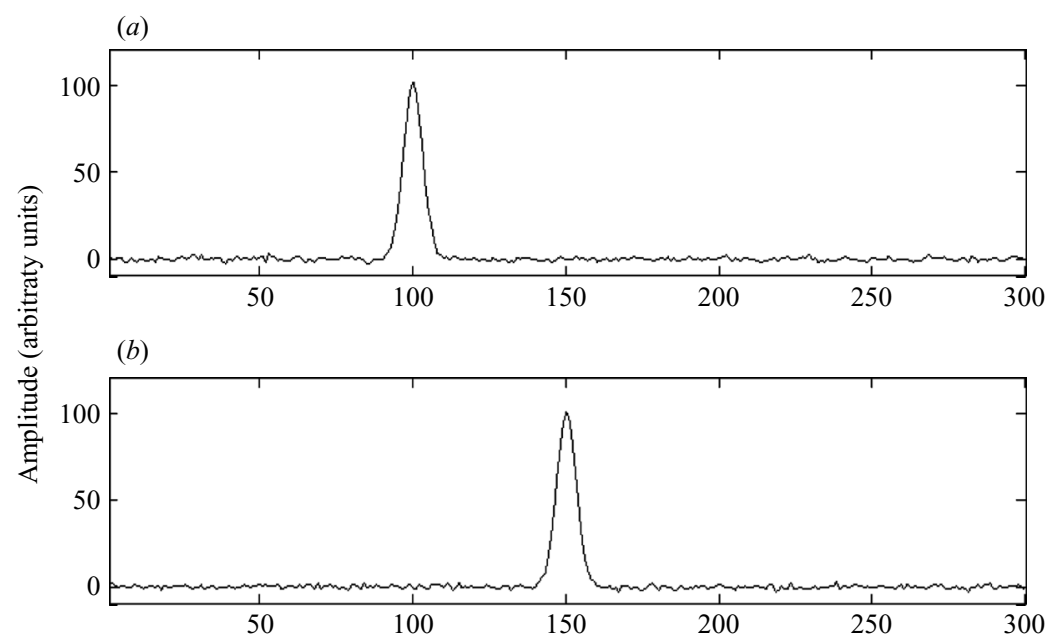

(c)

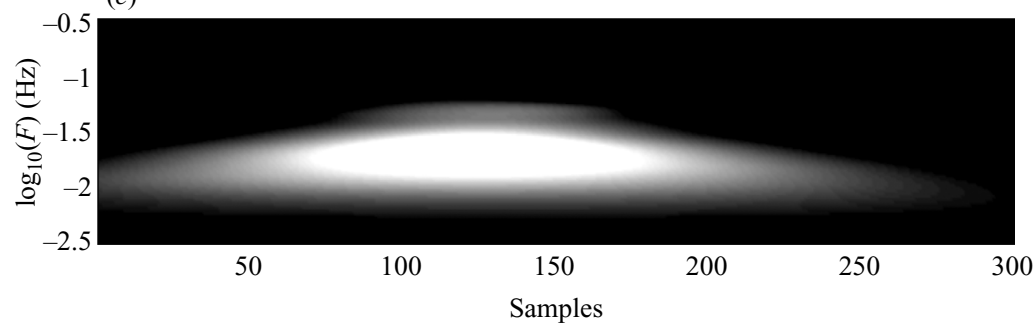

FIGURE 1. $(a, b)$ Smoothed wavelet cross-scalogram $\left|\left\langle w_{12}(r, t)\right\rangle\right|^{2}$ computed between two artificial 300-point signals with an arbitrary unitary sampling rate. The two peaks are located at sample 100 and 150 respectively so that the delay $\Delta t$ between the two is 50 samples. The maximum of the local coherence $(c)$ is located at sample 125 and at a frequency of about 0.02 that corresponds to $1 / \Delta t$.

the filter width and shape influences the coherence level and smoothness but does not change the peak location.

The selection of the events exhibiting large local coherence is accomplished by setting an appropriate threshold to trigger large amplitudes and deliver a sub-set of time instants and scales. The events selection is performed by a search of maxima within the subset of data exceeding the threshold. Therefore, the threshold is used only to limit the number of relative maxima that the tracking algorithm may select as true events. Owing to the presence of spurious maxima, the averaging process leads to a drop in the level with respect to the original signal. This effect is more pronounced for lower signal-to-noise ratios. However, in the present study the quantitative analysis of conditional averages is restricted to the time dependance of the signatures. As a consequence the drop in level is not a concern. It is, finally, stressed that the advantage in using a cross-wavelet transform with respect to computing a direct cross-correlation over a range of time delays lies mainly in the locality of the wavelet transform and the different resolution achievable at the different scales. This is the same as the reason why a wavelet transform is more reliable than a windowed Fourier transform (see e.g. Farge 1992 for more details).

An example clarifying the results to be expected is given in figure 1 where the wavelet coherence is computed between two artifical signals having peaks delayed an 


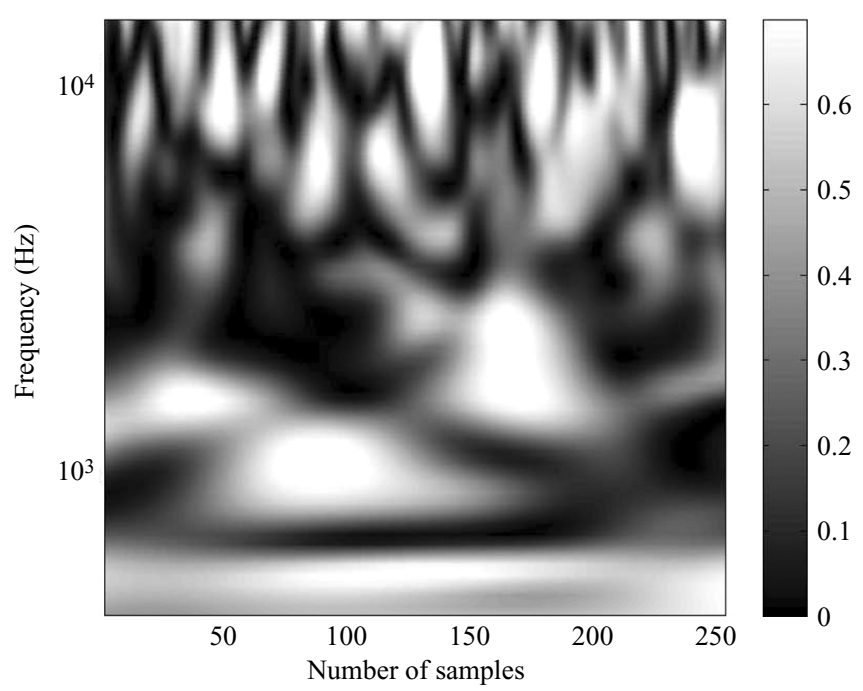

FIGURE 2. Example of wavelet coherence distribution computed from a portion of 256 samples of two pressure signals taken from the available databases.

arbitrary $\Delta t$. Note that in this example only the numerator of (2.4) is shown in order to avoid spurious effects due to very low amplitudes of the artificial wavelet coefficients. It is shown that a peak in the wavelet space is determined at a scale corresponding to about $1 / \Delta t$ and at a temporal location half way between the two events. An example of $R^{2}(r, t)$ obtained from a portion of two wall pressure signals from real experimental data is presented in figure 2 showing that, owing to the random nature of the signals, a larger number of peaks can be detected. In the present approach, since the maximum value of the wavelet coherence amplitude is 1 , the largest peaks are selected by fixing a trigger threshold at 0.8 , which is a good compromise between a high coherence level and a sufficiently large number of selections for the following statistical analyses. The subset of selected time instants corresponding to a local coherence overcoming the trigger level is denoted as $\left\{t_{0}^{i}\right\}$ where $i$ spans from 1 to $N_{s}$, $N_{s}$ being the number of selected events. In analogy with the artificial case presented in figure 1 , in the real situations the location of peaks at different wavelet scales should be attributed to events having different delay time. Thus, wavelet coefficients spanning across different frequency ranges can be excited depending on the shape and on the typical length scale of the physical structure generating the selected event. Therefore, the connection between frequency and time delay is much more complicated for real turbulence than for the artificial case reported in figure 1. Furthermore, it is important to clarify that, owing to the nature of the wavelet transform, high frequencies are better resolved than low ones and very long samples are needed to resolve low frequencies. Therefore no wavelet scale of interest is specified a priori and the selected events are not characterized in terms of their frequency. For the sake of accuracy, the only quantity retrieved from the events selection procedure is the time of appearance of the selected peaks, stored within the set $\left\{t_{0}^{i}\right\}$, while the frequency content is ignored.

Based on this background, a statistical analysis of the measured signals conditioned on the educed events has been carried out. The statistical quantities which are retrieved are the following: 
Waiting time statistics. The statistics of the time delay between two consecutive events is computed from the set $\left\{t_{0}^{i+1}-t_{0}^{i}\right\}$. As will be clarified below, this analysis is useful for its subsequent physical and statistical implications.

Statistics of the pressure magnitude. With reference to the original signal pair $p_{1}(t)$ and $p_{2}(t)$, the probability density functions (PDFs) of the pressure amplitudes corresponding to the triggered events, $p_{1}\left(t_{0}^{i}\right)$ and $p_{2}\left(t_{0}^{i}\right)$, are computed and compared to the statistics of the original signals.

Ensemble averages of the pressure signals. Conditional ensemble averages of the original time signals can be computed and the averaged time signature of the pressure events most likely to be responsible for the large correlations are extracted. The temporal averaging of the pressure signals is performed over all the signal segments (of arbitrary width) centred in the selected time instants $t_{0}^{i}$. For simplicity the time variable of the averaged pressure signature is denoted by $t$. The ensemble-averaging procedure can then be formalized as follows:

$$
\left\langle p_{1}(t)\right\rangle=\frac{1}{N_{s}} \sum_{i=1}^{N_{s}} p_{1}\left(t-t_{0}^{i}\right),
$$

where $\left\langle p_{1}(t)\right\rangle$ denotes the resulting averaged pressure signal, and, as pointed out above, the time $t$ extends over an appropriate interval.

The procedure of (2.8) is also applied to the other available signal $p_{2}$, from which the wavelet coherence is computed, generating another averaged signature $\left\langle p_{2}(t)\right\rangle$. In order to clarify the physical meaning of the averaged results, the procedure has been applied to two artificial signals generated $a d$ hoc by superimposing a large number of artificial peaks distributed randomly in time and imposing a fixed phase shift between the two signals. A segment of the generated signals is shown in figure 3 together with the resulting averaged signatures. The arrows in figure 3(a) qualitatively point to the set of events selected showing that a number of events correlated with the background noise can be included in the averaging procedure. Note that the overall length of the artificial signal is $5 \times 10^{6}$ samples while the reported segment includes only $10^{3}$ samples; thus the reported behaviour is rather qualitative. However, as shown in the bottom plot of figure $3(b)$, the background noise effects are averaged out after the ensemble-averaging procedure and the method correctly retrieves the shape of the correlated events (i.e. the bumps) as well as the phase between them. As a further test, the technique has been applied to pairs of uncorrelated signals (e.g. obtained through a random number generator or by considering two signals not acquired simultaneously) showing that non-zero signatures could not be obtained. An example of such an application is shown in figure 4.

When the procedure is applied to signals from real flows, in analogy with the examples of figures 1 and 3, a time delay between the two averaged signatures might be detected if a unique shape of the pressure events responsible for the local correlation peaks is statistically relevant. From the physical viewpoint, whenever the signatures are non-zero, it is possible to address, with the aid of existing conceptual models, suitable interpretations of the results in order to clarify the physical nature of the selected events.

Spectral features. It is known that the wavelet transform can be used to reproduce standard Fourier quantities by integrating in time the whole set of wavelet coefficients (see e.g. Onorato et al. 1997 for examples, details and theoretical issues on this aspect). Conditional spectral quantities can therefore be computed by considering only the subset of selected wavelet events. This procedure will be applied to compute 

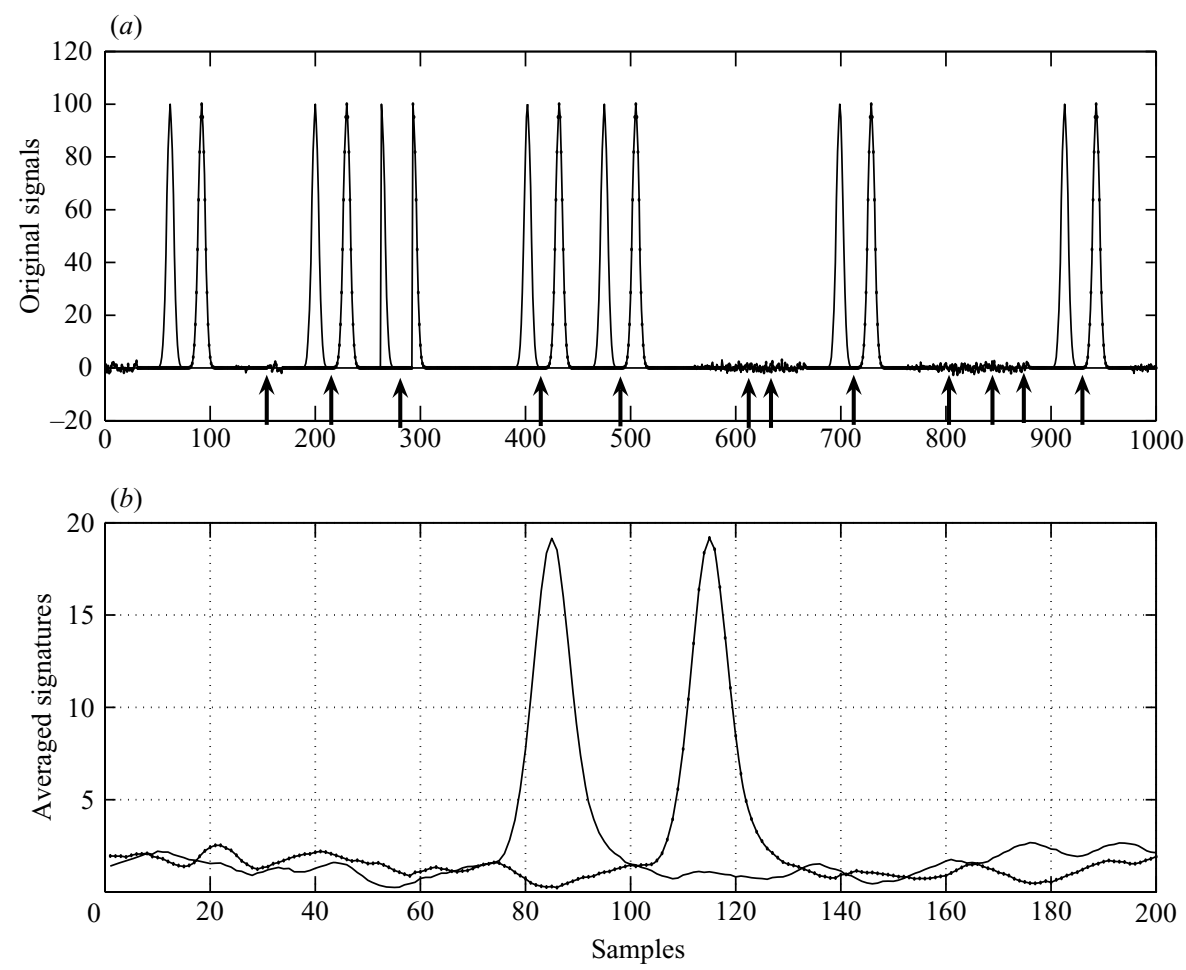

FIGURE 3. An example of the application of the averaging technique to two artificial signals (solid line and solid-dotted line of $(a)$ ) obtained by superimposing large Gaussian peaks on small random fluctuations. The actual length of the signals is about $5 \times 10^{6}$ samples and the imposed shift between two consecutive peaks is 30 samples. The averaging procedure is conducted by fixing a window of 200 samples centred upon the selected events. The resulting averaged signatures are shown in $(b)$. The separation between the averaged peaks is exactly 30 samples and the shape of the original peaks is also correctly reproduced. The arrows in (a), point to the timing of the selected events.

a conditional coherence function $\tilde{\gamma}(\omega)$ obtained by integrating the quantity $R^{2}(r, t)$ over a time span around $t_{0}^{i}$. Note that in the computation of $\tilde{\gamma}(\omega)$ the selection of the wavelet set is accomplished only on the basis of the selected time instants whereas the whole frequency content of each selected event is accounted for.

\section{The analysed databases}

As stated in the introduction, wavelet analyses have been carried out in equilibrium boudary layers. Therefore, in the present study, attention is drawn to non-equilibrium turbulent boundary layers. Two such databases from experimental low-Mach-number $(M \sim 0.1-0.15)$ studies are available. They are representative of realistic flow conditions with respect to practical applications. In particular, high Reynolds numbers and low non-vanishing pressure gradients are obtained in both cases.

The database named UR3 has been collected in the aerodynamic laboratory of DIMI at the University Roma Tre (Italy). Measurements were taken by a microphone pair flush mounted at the wall of a shallow cavity installed within a low-speed, not acoustically treated wind tunnel. The step height was fixed to $15 \mathrm{~mm}$ while the cavity length was $640 \mathrm{~mm}$. The cavity width was sufficiently large to consider the flow statistics as two-dimensional in the symmetry plane. Measurements were carried out 

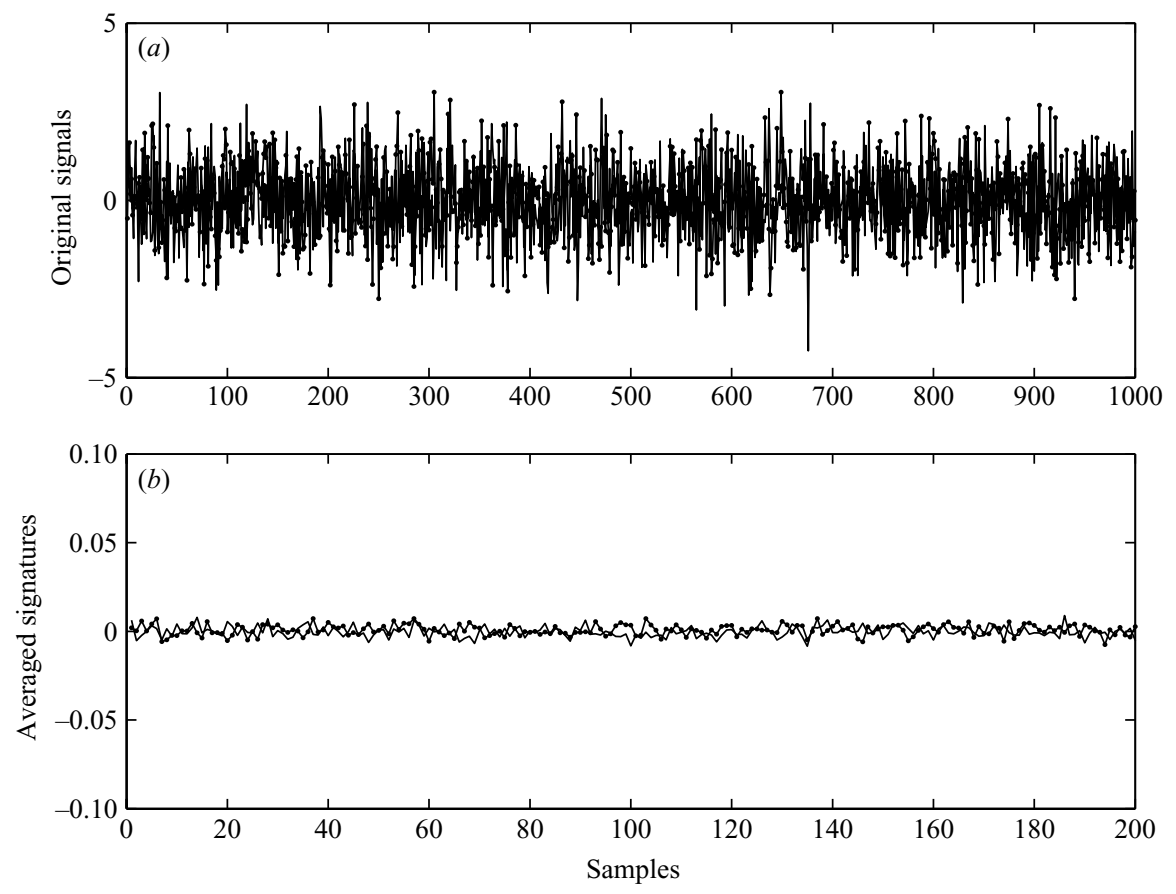

Figure 4. As figure 3 but showing two signals obtained from a random number generator (signals have Gaussian statistics with zero mean and unitary standard deviation).

using two microphones flush mounted at the cavity bottom wall. Two $1 / 8$ in. Brüel $\&$ Kjær 4138 microphones were used. Small diameter pinholes $(1 \mathrm{~mm})$ were used to connect the microphone cavity to the wall surface. The pinhole helps to minimize spatial averaging effects on the wall pressure fluctuation measurements. It has been checked that the micophone cavities generate no resonances in the frequency range of interest. Details about the experiment are given in Camussi et al. (2006a) and Camussi, Guj \& Ragni (2006b). Here we only mention that about $10^{5}$ samples have been acquired by each microphone at a sampling frequency of $20 \mathrm{kHz}$. The turbulent boundary layer displacement thickness was about $4 \mathrm{~mm}$ while the free-stream velocity spanned from 30 to $50 \mathrm{~m} \mathrm{~s}^{-1}$. The Reynolds number based on the step height and the free-stream velocity ranged from about $10^{4}$ to $5 \times 10^{4}$. Measurements have been conducted at several positions along the cavity floor while the separation between the microphones, aligned in the streamwise direction, was fixed to $25 \mathrm{~mm}$. For the sake of clarity, we consider only pressure signals obtained in the central region of the cavity where effects of the upstream and downstream steps are minimized though the boundary layer cannot be considered to be in equilibrium conditions. A sketch of the geometry considered and of the flow physics is shown in figure 5. It should be pointed out that the wind tunnel at the University Roma Tre is not anechoic and the background noise has been found to be related to both the blade passing frequency of the fan and to random excitation (details are given in Camussi et al. 2000). As will be shown later, the low signal-to-noise ratio and the limited number of samples acquired affect the statistical convergence of the results.

The analysis is extended by considering a database obtained from acoustic measurements performed within the anechoic wind tunnel of the Acoustics Centre of the Ecole Centrale of Lyon (hereafter denoted the ECL database). Wall pressure 


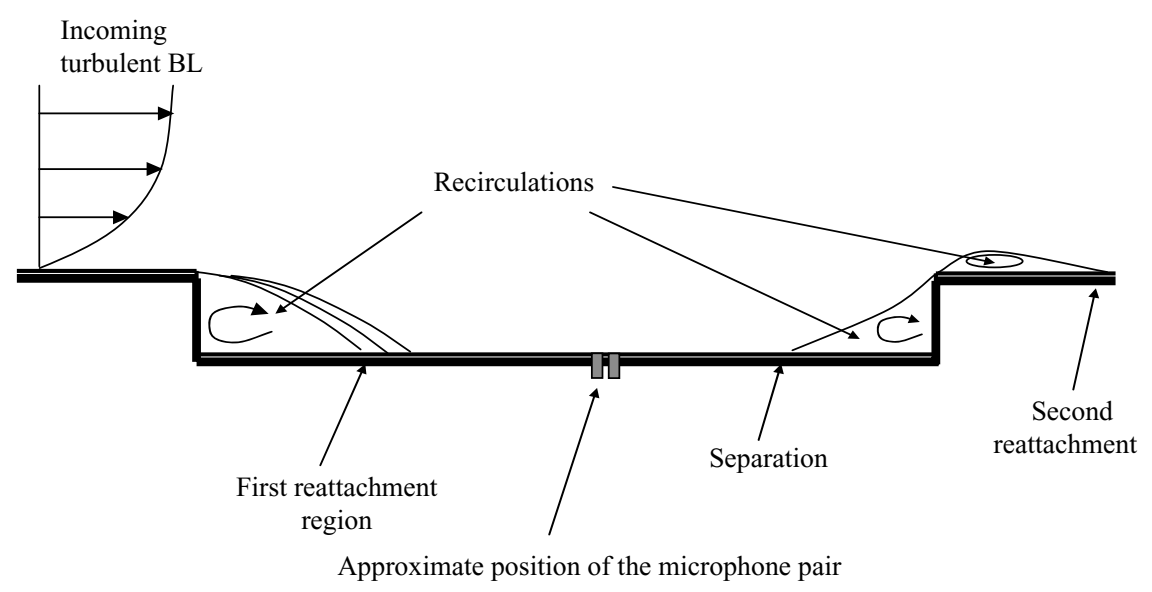

FIGURE 5. Sketch of the UR3 set-up showing the main recirculating flow regions.

fluctuations have been measured at the wall of a turbulent boundary layer in a channel which was geometrically modified to achieve a weak pressure gradient. The test section roof was made with a steel plate $\left(0.5 \times 4 \mathrm{~m}^{2}\right)$. Its thickness was $1.5 \mathrm{~mm}$ and 15 thread rods could be adjusted to modify the shape of a special adjustable roof. The test section was made airtight by a foam rubber joint.

The mean pressure gradients along the wind tunnel that were generated by adjusting the shape of this roof were measured with twenty-three static pressure pinholes made in the wind tunnel roof. Hot-wire measurements were also conducted in order to characterize the boundary layer velocity profiles while the wall shear stress was measured with a Preston tube and a Weiser probe. More details about the experimental set-up are given in Robert (2002). The displacement thickness at the measurement position was about $7.4 \mathrm{~mm}$ while the inlet free-stream velocity was set to $50 \mathrm{~m} \mathrm{~s}^{-1}$. The velocity at the minimum cross-section was increased by about $50 \%$ as an effect of the convergent roof and the maximum gradient of the pressure coefficient was of the order of $2 \mathrm{~m}^{-1}$. The wall pressure measurements were made using two $1 / 8$ in. Brüel \& Kjær microphones mounted on a traverse table embedded in the wall and made of two eccentric disks. Hence, an arbitrary displacement between the first microphone located at the centre of the main disk and a second microphone located on the smaller disk, was allowed. Separation distances between the microphones varied from 7.5 to $95 \mathrm{~mm}$ in all directions. In this experiment, no pinhole was used and the diameter of the microphones was about 200 viscous units. A filtering effect was therefore present at high frequencies since, as suggested by Schewe (1983), a transducer diameter $d^{+}<19$ is needed to resolve all essential wall pressure fluctuations. To account for this lack of resolution, Corcos (1963b) proposed a correction factor for the autospectra in terms of angular frequency, microphone radius and convection velocity. Schewe (1983) experimentally determined the Corcos correction to be adequate for $\omega d / U<4$. In the present cases, it was found that the frequency limit beyond which the Corcos correction is greater than $3 \mathrm{~dB}$ corresponded to about $5 \mathrm{kHz}$. Thus only the high-frequency range of the spectra is affected by the filtering effect.

The microphone signals were acquired in the time domain by an HP 3567A analyzer (Paragon), and about $10^{6}$ samples were acquired from each channel at a sampling frequency of $32768 \mathrm{~Hz}$. Several microphone locations are analysed below and the selected configurations will be specified when needed. A sketch of the geometry of the ECL experiment is given in figure 6. 


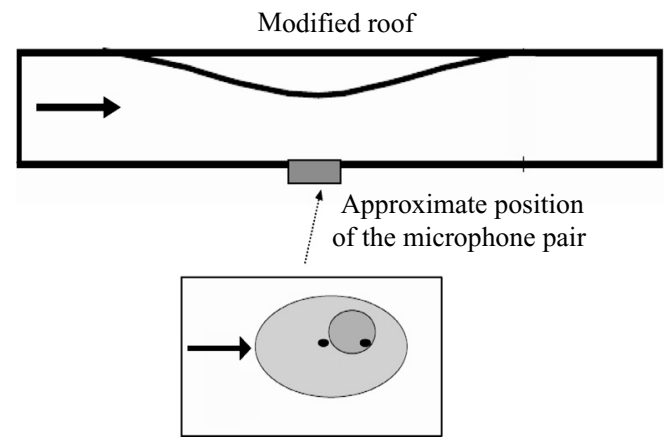

FIGURE 6. Sketch of the ECL set-up. The arrows indicate the direction of the inlet velocity.

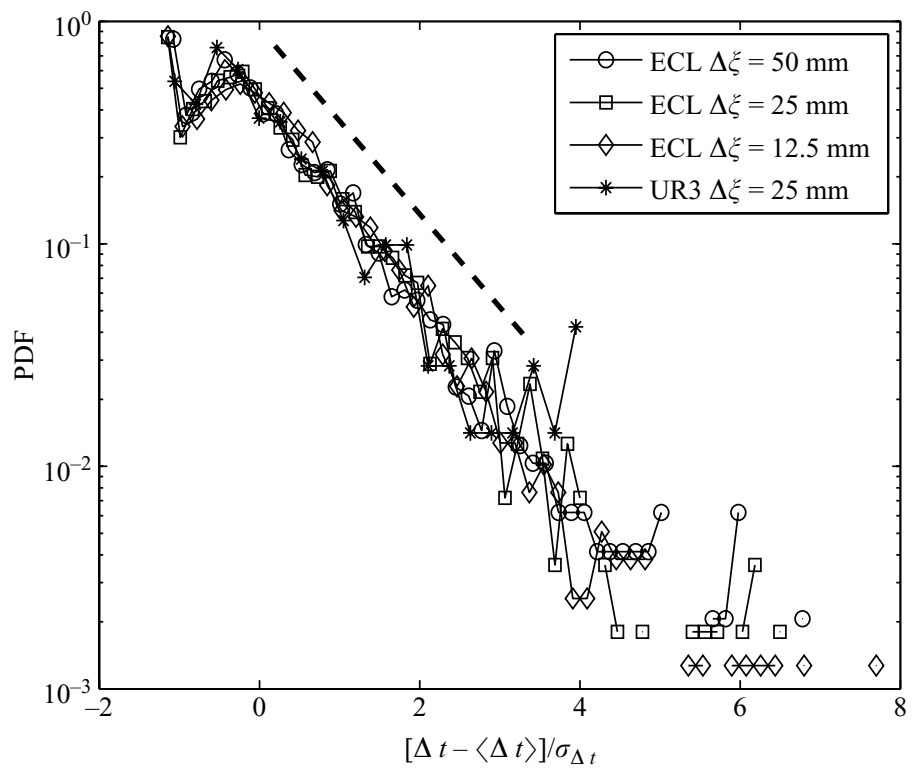

FIGURE 7. Semi-logarithmic plot of the PDFs of the time separation between successive events computed for different flow conditions. The dashed line denotes a pure exponential decay.

\section{Results}

The main results consist of the statistical indicators which have been defined in $\S 2$. It should be stressed that, for both databases, the number of events selected with the tracking procedure described in $\S 2$ is more than two orders of magnitude lower than the total number of samples. In the ECL database, the number of samples available is sufficiently large to ensure the statistical convergence of the selected event analysis. Conversely, because of the more limited number of samples, statistical convergence is not always achieved in the UR3 data; thus in some cases, only the ECL data will be considered.

In order to assess the statistical independence of the selected events, the statistics of the time separation between successive events is computed. The examples reported in figure 7 show a good collapse of the PDFs obtained from all data sets. As expected for statistically independent events, log-Poisson PDFs are observed (Feller 1968); the linear trend in the semi-log representation of figure 7 clearly indicates an exponential 


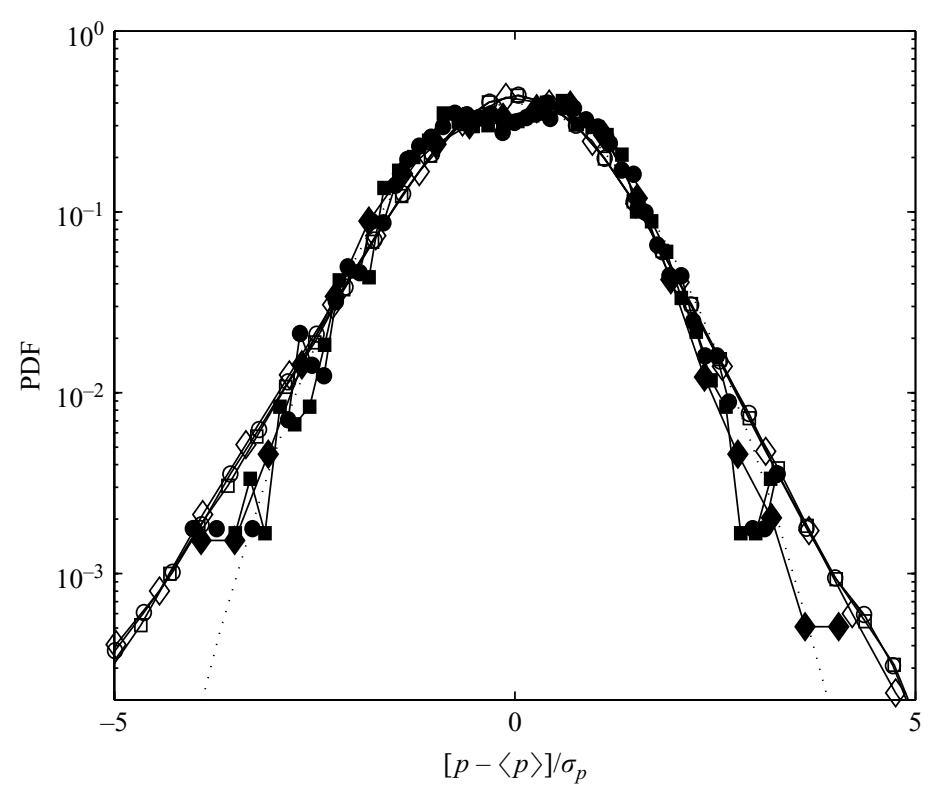

FIGURE 8. PDF of original pressure signals (open symbols) and selected pressure events (filled symbols). Pressure signals are taken from the ECL database and different symbols (squares, circles, diamonds) correspond to different separations between microphones at the wall. The dotted line represents a reference Gaussian curve having zero mean and unitary standard deviation. In all cases, only the signal retrieved from the transducer located upstream is considered.

decay law. This behaviour demonstrates that the educed events are not affected by periodic fluctuations or other spurious effects due to the background noise. The curves reported are strikingly similar to analogous results obtained from the analysis of the waiting-time statistics of coherent structures in turbulent flows (see e.g. Chainais, Abry \& Pinton 1999; Camussi \& Di Felice 2006). It can be argued that the pressure events educed therein are also correlated with organized structures embedded within the turbulent boundary layer and convected downstream. Further clarification of the physical nature of such structures will be given below.

When the PDFs of the wall pressure fluctuations are computed, symmetric nonGaussian curves with exponential tails are expected as an effect of the organized structures present within the boundary layer (see e.g. Kim 1989; Lamballais, Lesieur $\&$ Metais 1997). It is interesting to identify the contribution of the selected events to the overall statistical properties of the original pressure signals by computing the PDFs of the pressure amplitude corresponding to the timing $t_{0}^{i}$ of the selected events. Owing to the limited amount of available conditioned data, the analysis is conducted for the ECL database only. Examples of the PDFs obtained from the original and conditional pressure data sets are presented in figure 8 where a Gaussian curve is also included for reference. On one hand, the PDFs of the original signals exhibit the expected exponential tails with a symmetric distribution around the mean value. On the other hand, the conditional data are closer to a Gaussian PDF even though the comparison fails for large deviations from the mean value because of the lack of statistical convergence. This behaviour is due to the fact that, as was shown in $\S 2$, a selected time $t_{0}$ is located between the times of appearance of the events which are correlated with each other and which are presumed to be induced by organized 

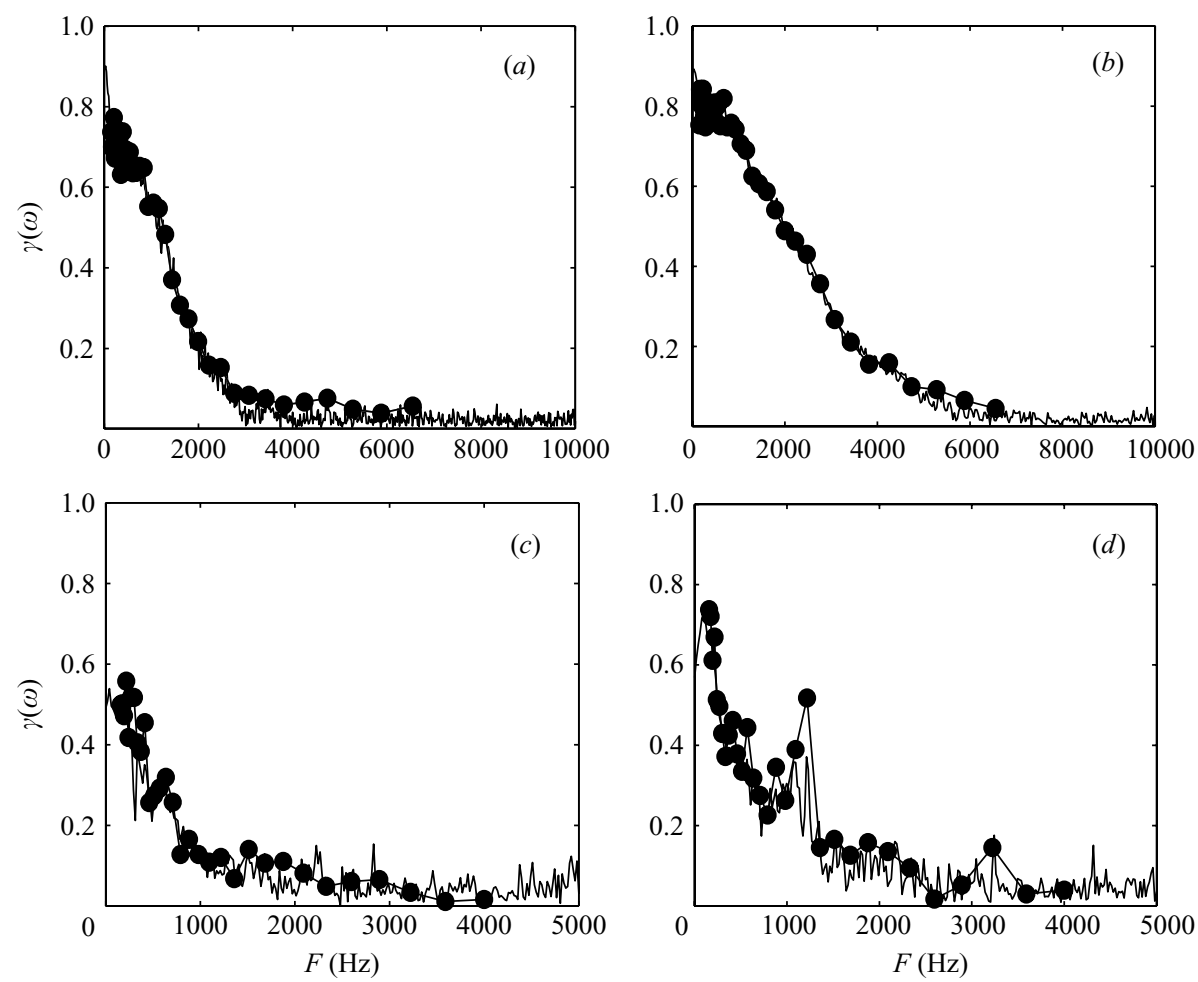

Figure 9. Coherence function computed with the standard Fourier-based approach (solid line, no symbols) compared with the conditional counterpart (filled circles). (a) ECL database with $\Delta \xi=50 \mathrm{~mm} ;(b)$ ECL database with $\Delta \xi=25 \mathrm{~mm} ;(c)$ UR3 data for free-stream velocity $30 \mathrm{~m} \mathrm{~s}^{-1} ;(d)$ UR3 data for free-stream velocity.

structures (see figure 1). Note that a random selection of a subset of amplitudes from an original pressure signal leads to a PDF which has exponential tails, thus demonstrating that the Gaussian nature of the conditional PDF has a physical meaning. This is a consequence of the fact that the sequence of events selected by the wavelet criterion is not purely random. The conditional PDFs also show a quasi bi-modal distribution in the sense that the highest probability does not coincide with the mean value but corresponds to pressure magnitudes lower and higher than the mean value of about one standard deviation.

Examples of the conditional coherence function computed from both data sets following the procedure described in $\S 2$ are plotted on figure 9. In these plots, the Fourier equivalent is also shown for comparison and a very good agreement is observed. It should be stressed that if the Fourier coherence had been computed with the same number of samples as for the coherence $\tilde{\gamma}(\omega)$, the result would have been much noisier because of the lack of statistical convergence.

Figure 9 demonstrates that the selection procedure is able to track events that are responsible for the observed Fourier coherence. This outcome is quite important from the theoretical viewpoint since, as was pointed out above, the coherence function plays a fundamental role in the theoretical models. It has been verified that the autospectra could also be correctly reconstructed using only the selected set of events. These results are not reported here since they are less meaningful from the theoretical viewpoint. 


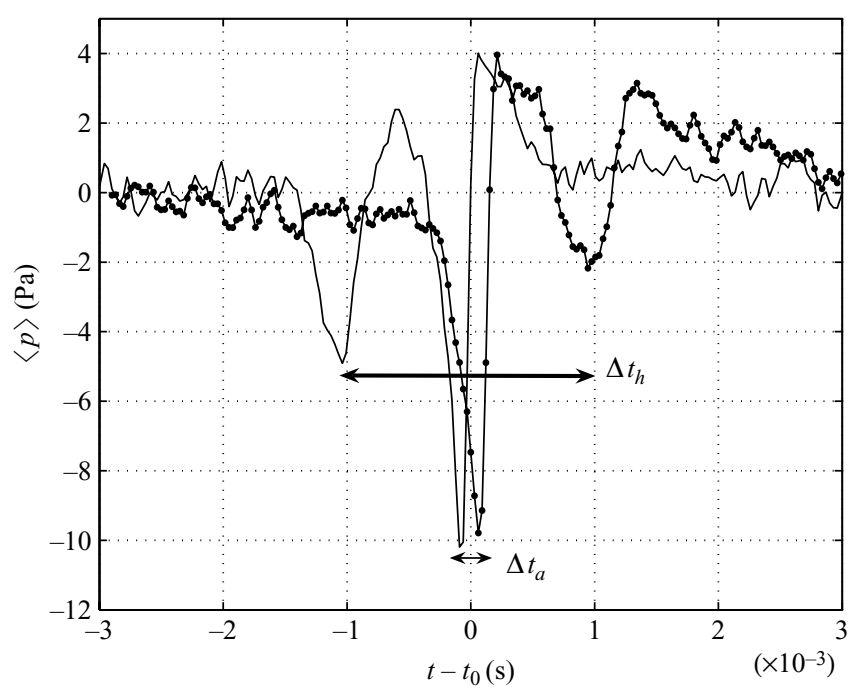

FIGURE 10. Averaged time signature of the wall pressure fluctuations conditioned on the wavelet coherence events. Pressure signals belong to the ECL database and correspond to a pressure transducer separation $\Delta \xi$ of $50 \mathrm{~mm}$ in the streamwise direction. Solid line corresponds to the pressure transducer located upstream, solid line with dots to the transducer downstream. $\Delta t_{h}$ is the time delay between events associated with hydrodynamic perturbations while $\Delta t_{a}$ is the analogous for acoustic events.

In order to understand the physical nature of the selected events responsible for the observed statistics, conditional ensemble averages of the pressure signals in the physical domain have been computed according to the procedure outlined in $\S 2$.

An example of the averaged pressure time signature obtained from the ECL database, is reported in figure 10. For each pressure transducer, two pressure drops are observed corresponding to different temporal separations, shown in the figure by the arrows. On account of the separation distance between the transducers and of the time delay measured between corresponding negative peaks, it is possible to compute two phase velocities. For the smallest time delay, denoted in the figure $\Delta t_{a}$, the phase velocity is close to the speed of sound, though the exact value cannot be retrieved owing to the limited sampling rate adopted. Therefore, the sharp pressure drops associated with $\Delta t_{a}$ can be interpreted as induced by unsteady compressibility or nearfield acoustic effects. These drops could possibly be related to the low wavenumbers of the subconvective region observed in classical wavenumber-frequency spectra (Bull 1996). The largest time delay, denoted $\Delta t_{h}$, corresponds instead to a convection velocity which is a fraction, about $30 \%$, of the free-stream velocity, thus representing the effect of hydrodynamic perturbations.

From the technical viewpoint it has been checked that the observed behaviour does not depend on the choice of the threshold level used to selected the events, except for a weak variation of the signal-to-background noise ratio. It has been also verified that similar results are obtained when only the smoothed wavelet cross-spectrum, i.e. the numerator of $R^{2}(r, t)$ defined in (2.4), is considered, thus suggesting that the peaks in the wavelet cross-spectrum are mostly due to physical correlated large-amplitude events rather than to small amplitude or even spurious effects.

The acoustic propagation appears much clearer when the microphones are separated in the spanwise direction, as shown on figure 11. In this case the hydrodynamic 


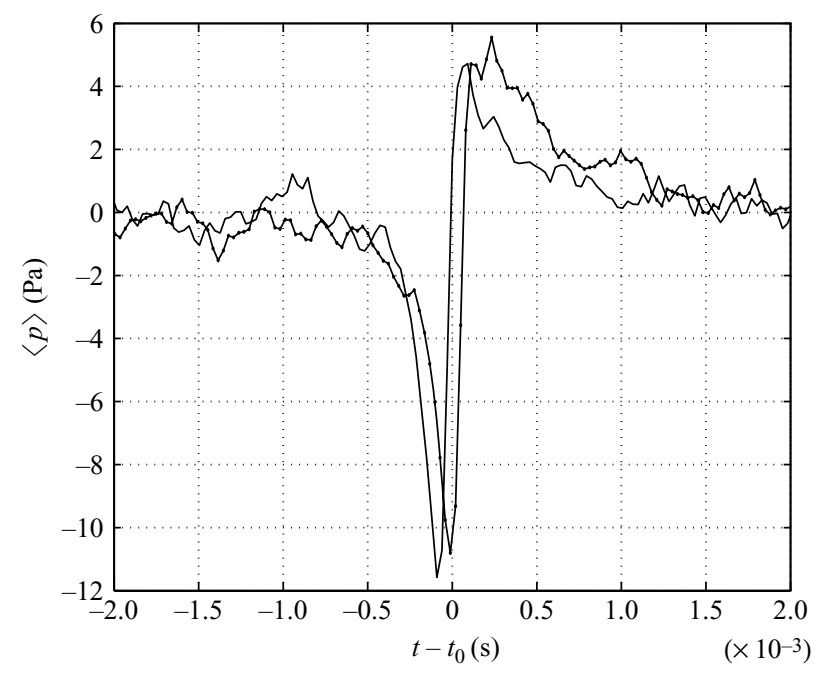

FiguRE 11. As figure 10 but for two probes separated in the spanwise direction.

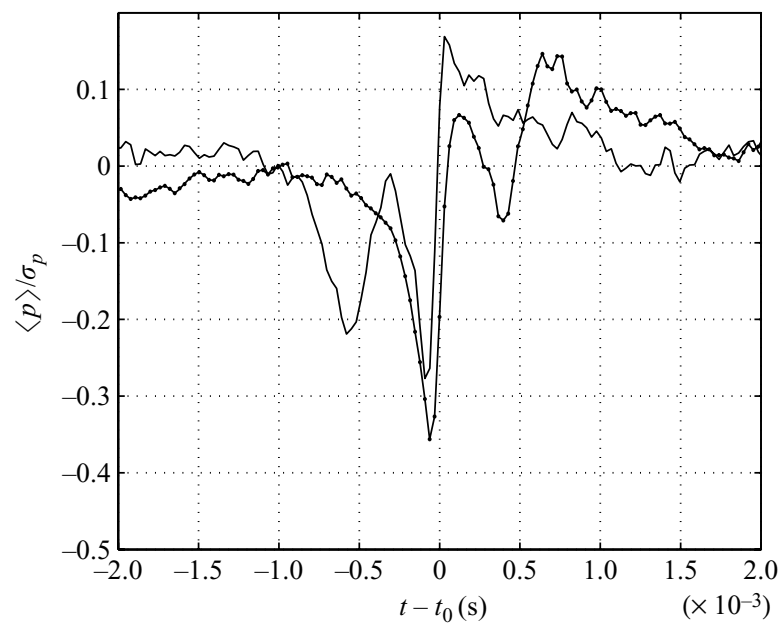

FIGURE 12. As figure 10 but for a pressure transducer separation $\Delta \xi$ of $25 \mathrm{~mm}$ in the streamwise direction. The averaged pressure amplitudes are normalized with respect to the standard deviation of the original corresponding signals.

contribution due to the mean stream advection weakens and only one pressure drop having a very small time delay is revealed.

When different streamwise separations are considered (figures 12 and 13), it can be observed that the hydrodynamic time delay varies whereas the compressibility effects, being associated to a very large propagation velocity, remain concentrated close to the time origin. The hydrodynamic time delay variation for the different streamwise separations considered leads to a constant convection velocity which is found to be, again, about $30 \%$ of the free-stream velocity.

Examples of results obtained from the UR3 data base are reported in figures 14 and 15 corresponding to a free-stream velocity of 30 and $50 \mathrm{~m} \mathrm{~s}^{-1}$ respectively. Although the quality of the results is flawed by the low signal-to-noise ratio, an 


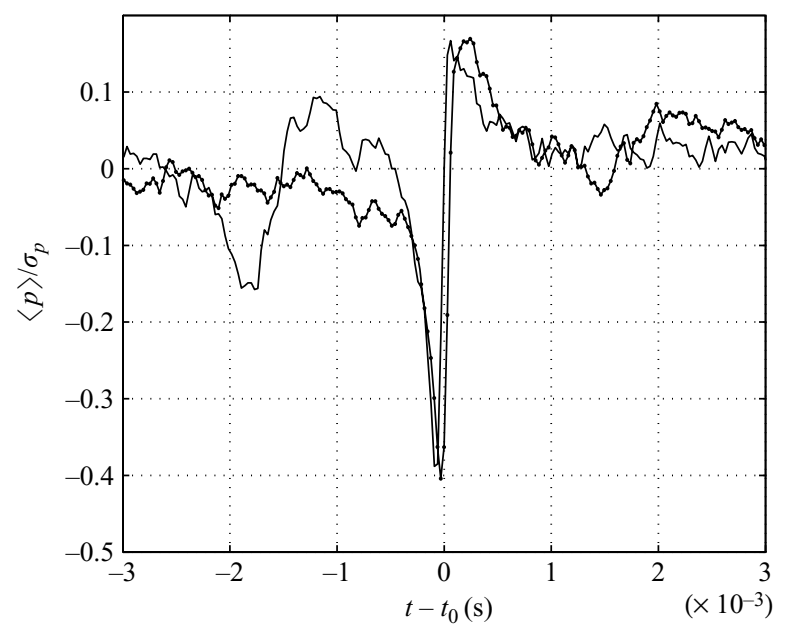

FIGURE 13. As figure 10 but for a pressure transducer separation $\Delta \xi$ of $90 \mathrm{~mm}$ in the streamwise direction. The averaged pressure amplitudes are normalized with respect to the standard deviation of the original corresponding signals.

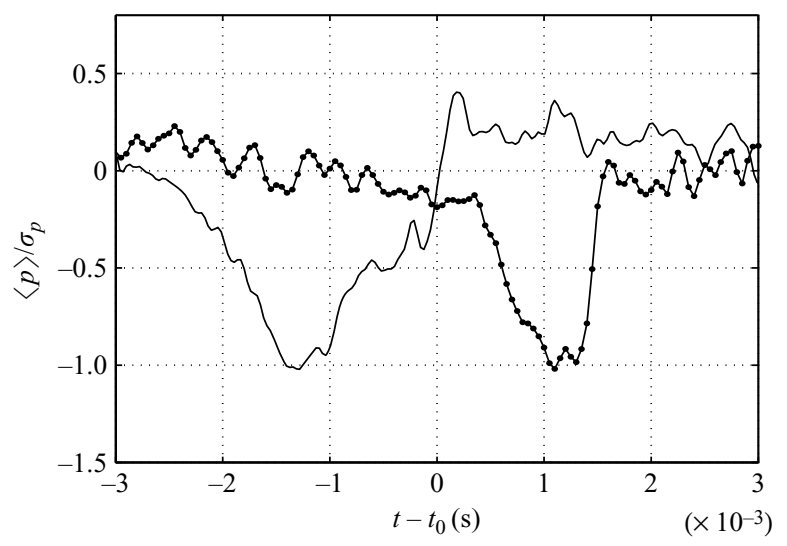

FIGURE 14. Averaged time signatures of the wall pressure fluctuations computed from pressure signals from the UR3 database. Solid line corresponds to the pressure transducer located upstream, solid with line dots to the transducer downstream. The free-stream velocity is $U=30 \mathrm{~m} \mathrm{~s}^{-1}$.

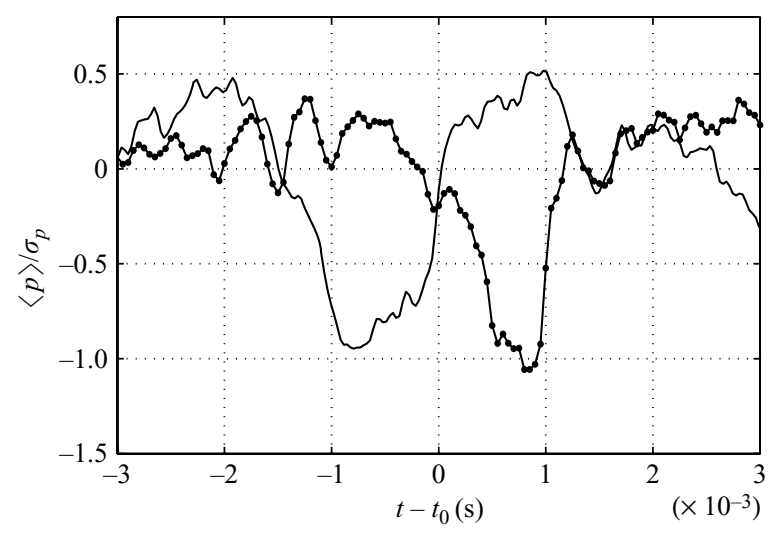

Figure 15. As figure 14 but for $U=50 \mathrm{~m} \mathrm{~s}^{-1}$. 
averaged signature can be observed. From a comparative analysis of the two figures it is possible to confirm the hydrodynamic nature of the pressure events since the time delay changes according to the different free-stream velocities. A convection velocity of about $30 \%$ of the inflow mean velocity is again found in both cases, in agreement with results from the ECL database. Also, the shape of the hydrodynamic signatures, though only qualitatively, is the same as the ECL cases and still can be interpreted as a pressure drop. Conversely, it should be stressed that the unsteady compressibility effects obtained from the analysis of the ECL data are no longer observed. Possibly this is due to that fact that in the UR3 facility, the sampling rate adopted is too low $(20000 \mathrm{~Hz})$ to resolve such rapid pressure variations. Indeed, as the separation between the two microphones is $25 \mathrm{~mm}$, a pressure perturbation moving at a velocity of about $340 \mathrm{~m} \mathrm{~s}^{-1}$, should lead to a time delay of less than two sampling intervals. Furthermore, the absence of an acoustic treatment and the very high background noise level, which has a random nature and non-homogeneous spatial distribution, dominates the effect of the pressure waves moving in the streamwise direction.

A accurate analysis of the results found, in particular of those from the ECL database, reveals that the averaged pressure signatures due to hydrodynamic effects are composed of a large negative pressure drop coupled with a weaker positive bump. This behaviour was reflected in the pressure conditional statistics which exhibited a bi-modal distribution (see figure 8). This was an effect of the negative-positive pressure magnitudes (or vice versa) which can be induced by accelerated-decelerated motions within the turbulent boundary layer.

On one hand, when the separation between the microphones is too small, the weaker positive bump is no longer detected since the acoustic signature contaminates the hydrodynamic one. On the other hand, for sufficiently large separations and high signal-to-noise ratios (see e.g. the case corresponding to $0.05 \mathrm{~m}$ of figure 10) the positive bump is detectable even though its amplitude is lower than that of the negative pressure peak. The presence of a positive pressure bump coupled with a stronger negative pressure drop was also observed by Dhanak et al. (1997) who simulated numerically the pressure field induced at the wall by streamwise vortices. Similar features have been observed experimentally by Johansson et al. (1987). They also observed negative-positive pressure jumps which were identified as burst-sweep events. Analogous conclusions were drawn by Jayasundera, Casarella \& Russel (1996) through the investigation of experimental wall pressure and inflow velocity data and the application of coherent-structure identification techniques. They showed that the organized structures present within the turbulent boundary layer contain both ejection and sweep motions inducing positive and negative pressure events respectively. In the present cases, the negative pressure peaks prevail over the positive bumps, suggesting that the underlying nature of the educed events is sweep-type motions prevailing over bursts. Furthermore, the values of the hydrodynamic convection velocities found in the present investigation are lower than the convection velocities usually measured in wall flows and ascribed to flow structures belonging to the outer region of the turbulent boundary layer. This result suggests that here the sources of wall pressure originate from the deep boundary layer, where the mean flow is slower.

These interpretations are supported by the conditional results reported by Johansson et al. (1987) who correlated pressure negative peaks with VITA velocity events found in the buffer region of the boundary layer. More recently, Kim, Choi \& Sung (2002) attempted to correlate the wall pressure fluctuations with the streamwise vortices of a numerically simulated turbulent boundary layer. They suggest that the 
high negative wall pressure fluctuations are due to outward motion in the vicinity of the wall correlated with the presence of streamwise vortices.

Numerical simulations or simultaneous measurements of the wall pressure fluctuations and the flow field (e.g. using the particle image velocimetry technique) would be necessary to clarify the topological nature of the educed structures as well as their spatial location. This challenging task is left for future studies.

\section{Conclusions and final remarks}

Wall pressure fluctuations obtained in two different laboratory turbulent boundary layers have been analysed by the application of a cross-wavelet transform. The methodology used allows conditional statistics to be carried out by selecting events exhibiting strong localized coherence.

The conditional statistics have shown that the selected events are statistically independent since the PDFs of the time delay between successive events display a typical log-Poisson behaviour. Conversely the pressure magnitudes for the selected time set are close to Gaussian PDF, though exhibiting a bi-modal behaviour around the mean value as a consequence of highly probable coupled negative-positive pressure peaks.

From the spectral viewpoint, it is shown that the conditional coherence function coincides with its non-conditional Fourier equivalent. This means that the set of selected events, though composed of some data more than two orders of magnitude lower than that of the original signals, reflects correctly the original spectral properties which are relevant for the purpose of theoretical modelling.

The averaged pressure time signatures revealed the effect of both hydrodynamic and unsteady compressibility effects. These acoustic-like signatures are identified as sharp pressure variations induced in the vicinity of the structure where they move at a phase velocity close to the speed of sound. They are only detected if the time resolution is fine enough. In the hydrodynamic cases, the phase velocity is a fraction of the inflow free-stream velocity and corresponds to a convection velocity.

With the aid of previous experimental and numerical analyses, the hydrodynamic signatures have been interpreted as induced by near-wall-sweep type events which are known to be closely associated with the presence of streamwise vortices embedded within the turbulent flow and located in the near-wall region. The sweep-type motion therefore represents the major physical mechanism responsible for the observed conditional statistics and the main near-wall feature that has to be controlled if a manipulation of the wall pressure spectral properties, in particular of the coherence function, is to be accomplished.

The authors acknowledge support from EU under contracts AST4-CT-2005-012222 (PROBAND project) and G4RD-CT-2000-00223 (ENABLE project). R. C. also acknowledge support from Italian Ministry of Education, University and Research under a grant PRIN (2005). W. R. Graham and D. Leclercq are gratefully acknowledged for their careful reading of the draft manuscript and the useful comments and suggestions provided.

\section{REFERENCES}

Ahn, B.-K., Graham, W. R. \& Rizzi, S. A. 2004 Modelling unsteady wall pressures beneath turbulent boundary layers. NASA-AIAA 2004-2849. 
Bendat, J. S. \& Piersol A. G. 2000 Random Data: Analysis and Measurements Procedures, 3rd ed. Wiley.

BlaKe, W. K. 1986 Mechanics of Flow Induced Sound and Vibrations. Academic.

BRADSHAW, P. 1967 Inactive motion and pressure fluctuations in turbulent boundary layers. J. Fluid Mech. 30, 241-258.

Brungart, T. A., Lauchle, G. C., Deutsch, S. \& Riggs, E. T. 2002 Wall pressure fluctuations induced by separated/reattached channel flow. J. Sound Vib. 251, 558-577.

BuLL, M. K. 1996 Wall pressure fluctuations beneath turbulent layer: some reflections on forty years of research. J. Sound Vib. 190, 299-315.

CAmussi, R. \& GuJ, G. 1997 Orthonormal wavelet decomposition of turbulent flows: intermittency and coherent structures. J. Fluid Mech. 348, 177-199.

Camussi, R., Guj, G., Barbagallo, D. \& Prischich, D. 2000 Experimental characterization of the aeroacoustic behaviour of a low speed wind tunnel. AIAA Paper 2000-1986.

Camussi, R. \& Di Felice, F. 2006 Statistical properties of large scale spanwise structures in zero pressure gradient turbulent boundary layers. Phys. Fluids 18, 035108.

Camussi, R., Guj, G., Di Marco, A. \& Ragni, A. $2006 a$ Propagation of wall pressure perturbations in a large aspect-ratio shallow cavity. Exps. Fluids 40, 612-620.

Camussi, R, Guj, G. \& Ragni, A. $2006 b$ Wall pressure fluctuations induced by turbulent boundary layers over surface discontinuities. J. Sound Vib. 294, 177-204.

Chainais, P., Abry, P. \& Pinton, J. F. 1999 Intermittency and coherent structures in a turbulent flow: a wavelet analysis of joint pressure and velocity measurements. Phys. Fluids 11, 35243539.

Chase, D. M. 1980 Modeling the wave-vector frequency spectrum of turbulent boundary layer wall pressure. J. Sound Vib. 70, 29-68.

Corcos, G. M. 1963a The structure of the turbulent pressure field in boundary-layer flows. J. Fluid Mech. 18, 353-378.

Corcos, G. M. $1963 b$ Resolution of pressures in turbulence. J. Acous. Soc. Am. 35, 192-199.

Daubechies, I. 1992 Ten Lectures on Wavelet. CBMS-NSF Reg. Conf. Ser. Appl. Maths.

Dhanak, M. R. \& Dowling, A. P. 1995 On the pressure fluctuations induced by coherent vortex motion near a surface. AIAA Paper 95-2240.

Dhanak, M. R., Dowling, A. P. \& Si, C. 1997 Coherent vortex model for surface pressure fluctuations induced by the wall region of a turbulent boundary layer. Phys. Fluids 9, 27162731.

Efimtsov, B. M. 1986 Vibrations of a cylindrical panel in a field of turbulent pressure fluctuations. Sov. Phys. Acoust. 32, 336-337.

Farabee, T. M. \& Casarella, M. J. 1991 Spectral features of wall pressure fluctuations beneath turbulent boundary layers. Phys. Fluids A 3, 2410-2420.

FARGE, M. 1992 Wavelet transforms and their applications to turbulence. Annu. Rev. Fluid Mech. 24, 395-457.

Feller, W. 1968 An Introduction to Probability Theory and Its Applications, 3rd edn. Wiley.

Goupillaud, P., Grossmann, A. \& Morlet, J. 1984 Cycle-octave and related transforms in seismic signal analysis. Geoexploration 23, 85-102.

Graham, W. R. 1997 A comparison of models for the wavenumber-frequency spectrum of turbulent boundary layer pressures. J. Sound Vib. 206, 541-565.

Grinsted, A., Moore, J. C. \& Jevrejeva, S. 2004 Application of the cross wavelet transform and wavelet coherence to geophysical time series. Non Lin. Proc. Geophys. 11, 561-566.

Guj, G. \& CAmussi, R. 1999 Statistical analysis of local turbulent energy fluctuations, J. Fluid Mech. $382,1-26$.

Hudgins, L., Friebe, C. \& Mayer, M. 1993 Wavelet transforms and atmospheric turbulence. Phys. Rev. Lett. 71, 3279-3282.

Jayasundera, S., Casarella, M. \& Russel, S. 1996 Identification of coherent motions using wall pressure signatures. Tech. Rep. 19960918-036, Catholic Univ. of America, Washington DC. (available at http://handle.dtic.mil/100.2/ADA314537).

Jevrejeva, S., Moore, J. C. \& Grinsted, A. 2003 Influence of the arctic oscillation and El NinoSouthern Oscillation (ENSO) on ice conditions in the Baltic Sea: The wavelet approach. $J$. Geophys. Res. 108, 4677-4681. 
Johansson, A. V., Her, J.-Y. \& Haritonidis, J. H. 1987 On the generation of high-amplitude wall-pressure peaks in turbulent boundary layers and spots. J. Fluid Mech. 175, 119-142.

KIM, J. 1989 On the structure of pressure fluctuations in simulated turbulent channel flow. J. Fluid Mech. 205, 421-451.

KIm, J., ChOI, J. \& Sung, H. J. 2002 Relationship between wall pressure fluctuations and streamwise vortices in a turbulent boundary layer. Phys. Fluids A 14, 898-901.

Lamballais, E., Lesieur, M. \& Metais, O. 1997 Probability distribution functions and coherent structures in a turbulent channel. Phys. Rev. E 56, 6761-6766.

LEE, I. \& Sung, H. J. 2002 Multiple-arrayed pressure measurement for investigation of the unsteady flow structure of a reattaching shear layer. J. Fluid Mech. 463, 377-402.

LI, H. 1998 Identification of coherent structures in turbulent shear flow with wavelet correlation analysis. Trans. ASME: J. Fluids Engng 120, 778-785.

MaLlat, S. 1989 A theory for multiresolution signal decomposition: the wavelet representation. IEEE Trans. PAMI 11, 674-693.

Maraun, D. \& Kurths, J. 2004 Cross wavelet analysis: significance testing and pitfalls. Non lin. Proc. Geophys. 11, 505-514.

Onorato, M., Salvetti, M. V., Buresti G. \& Petagna, P. 1997 Application of a wavelet crosscorrelation analysis to DNS velocity signals. Eur. J. Mech. B/Fluids 16, 575-597.

Orlandi, P. \& JimeneZ, J. 1994 On the generation of turbulent wall friction. Phys. Fluids 6, 634-641.

Perry, A. E. \& Chong, M. S. 1982 On the mechanism of wall turbulence. J. Fluid Mech. 119, 173-217.

Poggie, J. \& Smiths, A. J. 1997 Wavelet analysis of wall-pressure fluctuations in a supersonic blunt fin flow. AIAA J. 35, 1597-1603.

Robert, G. 2002 Experimental data base for the pressure gradient effect. Rep. 3.2.1. UE Research Programme, Contract No. G4RD-CT-2000-00223.

SCHEwE, G. 1983 On the structure and the resolution of wall pressure fluctuations associated with turbulent boundary-layer flow. J. Fluid Mech. 134, 311-328.

Smol'yakov, A. V. \& TKachenko, V. M. 1991 Model of a field of pseudosonic turbulent wall pressures and experimental data. Sov. Phys. Acoust. 37, 627-631.

Torrence, C. \& Compo, G. P. 1998 Apractical guide to wavelet analysis. Bull Am. Met. Soc. 79, 61-78.

Torrence, C. \& Webster, P. J. 1999 Interdecadal changes in the ENSOMonsoon system. J. Climate 12, 2679-2690. 